\title{
Helicobacter pylori-Associated Upper Gastrointestinal Symptoms: FD or HpD?
}

\author{
Hidekazu Suzuki ${ }^{1}$
}

Published online: 1 April 2017

(c) Springer Science+Business Media New York 2017

The possible pathogenic and etiologic link between Helicobacter pylori (H. pylori) infection and dyspeptic symptom generation, extensively discussed in international fora, $[1,2]$ has been incorporated into the recent Rome IV classification [3] and guidelines [4, 5]. H. pylori-originated dyspeptic symptoms are currently classified as non-functional, organic disorders.

In this issue of Digestive Diseases and Sciences, Lee et al. reported a cross-sectional study [6], in which the authors concluded that $H$. pylori seropositivity is not an independent risk factor for upper gastrointestinal (GI) symptom generation. Their patient population included adult subjects older than 18 years who underwent upper GI endoscopy on the same day that serum pepsinogen and anti-H. pylori IgG assays were obtained. Of the 2275 subjects, $31.8 \%$ had at least one upper GI symptom, and $16.4 \%$ had multiple upper GI symptoms; the symptoms included globus sensation, odynophagia, chest discomfort, heartburn, dysphagia, postprandial fullness, early satiety, epigastric soreness, upper abdominal pain, nausea, and vomiting. Even after the exclusion of organic disorders, their subject population included functional esophageal, gastroduodenal, and other functional GI disorders. For example, among these symptoms, those that could be related to functional dyspepsia (FD) include postprandial fullness, early satiety, epigastric soreness, upper abdominal pain, and nausea. Conversely, other symptoms, such as globus sensation, odynophagia, chest discomfort,

Hidekazu Suzuki

hsuzuki.a6@keio.jp

1 Medical Education Center, Keio University School of Medicine, 35 Shinanomachi, Shinjuku-ku, Tokyo 160-8582, Japan heartburn, dysphagia, or vomiting, would suggest functional disorders other than FD. Among their symptompositive cohort, $58.5 \%$ were $H$. pylori-seropositive, whereas among their symptom-negative control group, $61.8 \%$ were $H$. pylori-seropositive, suggesting a higher positivity ( $\sim 60 \%$ ) of $H$. pylori infection in this population. Conversely, among their 1382 seropositive subjects, $30.6 \%$ had symptoms originating from the esophagus, stomach, duodenum, or other organs; among their 893 seronegative subjects, $33.6 \%$ also had some of those symptoms, suggesting a similar symptom positivity rate between the $H$. pylori-positive and negative cohorts. They also mentioned that the gastric corpus atrophy cohort was $H$. pyloriseronegative, suggesting that the patients either had autoimmune gastritis or had severe atrophic gastritis from spontaneously remitted $H$. pylori, both serum pepsinogen positive. Although they concluded that $H$. pylori is not an independent risk factor for upper GI symptoms, their cohorts (seropositive and symptom-positive groups) included patients with symptoms originating from $H$. pylori infection. To rigorously investigate the possible relation of some symptoms to $H$. pylori infection, symptomatic remission after the eradication of $H$. pylori is a logical endpoint that has been studied, as detailed below.

A recent meta-analysis by Zhao et al. [7] reported a statistically significant improvement in dyspepsia symptoms in their treatment group $(40.6 \%)$ but not in the control group $(34.0 \%)$. The number needed to treat (NNT) to consider eradication as effective was 15; nevertheless, it decreased to 9 when limited to studies of Asian populations. According to a double blind, randomized, placebocontrolled trial of $H$. pylori eradication for FD by Gwee et al. in a Singapore-based Asian population using intention-to-treat analyses [8], symptom resolution was observed in $24 \%$ of patients receiving active treatment and 
$7 \%$ receiving placebo; the difference in the proportion of patients with symptom resolution was statistically significant $(p=0.02,95 \%$ confidence interval $=1.1-17.7)$. Among patients with successful $H$. pylori eradication, the symptom resolution rate was $39 \%(10 / 26)$, and among patients in the placebo group who had persistent $H$. pylori infection, the rate was $3 \%(1 / 35)$. In their multivariate analysis, post-treatment $H$. pylori status was the only predictor of symptom resolution. They concluded that in contrast to Western populations, patients with FD in Asia would benefit from the $H$. pylori eradication therapy with as much as a 13-fold increased possibility of symptom resolution following eradication [8]. Our previous study in Keio University Hospital that examined 68 patients with dyspeptic symptoms with $H$. pylori infection also reported that after successful eradication of $H$. pylori, abdominal pain was significantly relieved, in contrast to patients with unsuccessful eradication, in whom no symptomatic relief was obtained [9]. When taken together, it is suggested that symptom relief after successful eradication of $H$. pyloripositive dyspepsia is significant and is specifically higher in Asian than in Western populations.

According to the recent Rome IV definition [3], the use of medications, such as nonsteroidal anti-inflammatory drugs (NSAID)s, or $H$. pylori infection (H. pylori-associated dyspepsia [HpD]) are categorized as secondary dyspepsia that is different from FD. Nonetheless, in the present study, $H$. pylori infection as well as NSAID use was included in the entire study population. To consider the treatment of upper GI symptoms, such as dyspepsia, a diagnostic workflow should be used to clarify the known etiology, including $H$. pylori infection or use of drugs, such as NSAIDs.

As known by most physicians, $H$. pylori-positive gastritis is associated with histological inflammation, including polymorphonuclear and mononuclear cell infiltration, microvascular congestion, mucosal atrophy, and intestinal metaplasia $[10,11]$. H. pylori infection is also associated with microscopic duodenitis that has been linked with dyspeptic symptoms $[12,13]$. Nevertheless, dyspepsia associated with $H$. pylori infection has been customarily included under the umbrella term of FD, although $H$. pylori infection is clearly a major cause of $H$. pylori-induced gastritis (diagnosed via histological analysis). We first highlighted the importance of HpD in 2011, refining the concept of HpD in subsequent publications [14, 15]. The Kyoto Global consensus [2] classified $H$. pylori-positive dyspepsia and established the novel disease entity $\mathrm{HpD}[1,14,15]$. In this Kyoto document, a $92.1 \%$ consensus was achieved for the statement that $\mathrm{HpD}$ is a distinct entity from FD [2]. At the same time, the consensus report also determined that $\mathrm{HpD}$ was diagnosed after patients had sustained symptomatic relief with a delay of at least 6 months after cure of the infection [2] that is attributed to the slow recovery from $H$. pylori gastritis, particularly the recovery from gastric mucosal atrophy. Therefore, symptomatic relief should be ascertained at least 6-12 months after eradication in order to avoid transient placebo effects. This new trend has then been included in the novel Rome IV definition [3] and Maastricht V/Florence consensus [5]. Here, the important point is that not all dyspepsia symptoms originate from $H$. pylori infection even in $H$. pylori-positive cohorts. According to a previous randomized controlled trial by Gwee et al. [8], only $30 \%$ of symptomatic relief had been obtained after successful $H$. pylori eradication, suggesting that the remaining $70 \%$ would be $H$. pylori-unrelated. Accordingly, dyspepsia associated with $H$. pylori gastritis (H. pylori-infected dyspepsia) is thought to reflect those with $\mathrm{HpD}$ (secondary dyspepsia) and $H$. pylori-unrelated dyspepsia. These data satisfy the consensus that individuals who do not achieve relief from dyspepsia symptoms after $H$. pylori eradication are diagnosed as FD.

Until now, a diagnostic biomarker that can accurately predict $\mathrm{HpD}$ prior to symptom assessment 6-12 months after eradication has not been available. Even after the global consensus defining $\operatorname{HpD}[2,3,5]$, its diagnosis is complicated due to the requirement that at least 6 months of observation after eradication are needed. Conversely, the existence of false-negative findings among the serological tests (pepsinogen I and II and anti-H. pylori IgG antibody) for the assessment of gastric cancer risk stratification is a key point that should be considered [2]. The serum pepsinogen I level and pepsinogen I:II ratio can be normal even with residual atrophic gastritis after $H$. pylori eradication, obscuring the previous $H$. pylori infection. Moreover, although the sensitivity of anti- $H$. pylori IgG antibody is $\sim 90 \%$, it can also have false-negative results. For the assessment of seropositivity or corpus atrophic gastritis, caution is needed particularly for the interpretation of pepsinogen and anti-H. pylori IgG antibody titers.

After the Kyoto consensus, the Japanese guidelines for FD [4], Rome IV criteria [3], and Maastricht V/Florence consensus [5] all stated the tests and treatment strategies before the diagnosis of FD can be confirmed. These guidelines need to be followed by practitioners so as to maximize accuracy with FD diagnosis.

\section{References}

1. Suzuki H, Mori H. Helicobacter pylori: Helicobacter pylori gastritis-a novel distinct disease entity. Nat Rev Gastroenterol Hepatol. 2015;12:556-557.

2. Sugano K, Tack J, Kuipers EJ, et al. Kyoto global consensus report on Helicobacter pylori gastritis. Gut. 2015;64:1353-1367. 
3. Stanghellini V, Chan FK, Hasler WL, et al. Gastroduodenal disorders. Gastroenterology. 2016;150:1380-1392.

4. Miwa H, Kusano M, Arisawa T, et al. Evidence-based clinical practice guidelines for functional dyspepsia. J Gastroenterol. 2015;50:125-139.

5. Malfertheiner P, Megraud F, O'Morain CA, et al. European Helicobacter and Microbiota Study Group and consensus panel management of Helicobacter pylori infection-the Maastricht V/Florence consensus report. Gut. 2017;66:6-30.

6. Lee SP, Lee S-Y, Kim JH, Sung I-K, Park HS, Lee CSS. Factors related to upper gastrointestinal symptom generation in 2,275 Helicobacter pylori seroprevalent adults. Dig. Dis. Sci. (Epub ahead of print). doi:10.1007/s10620-017-4529-7.

7. Zhao B, Zhao J, Cheng WF, et al. Efficacy of Helicobacter pylori eradication therapy on functional dyspepsia: a meta-analysis of randomized controlled studies with 12-month follow-up. J Clin Gastroenterol. 2014;48:241-247.

8. Gwee KA, Teng L, Wong RK, Ho KY, Sutedja DS, Yeoh KG. The response of Asian patients with functional dyspepsia to eradication of Helicobacter pylori infection. Eur J Gastroenterol Hepatol. 2009;21:417-424.

9. Suzuki H, Masaoka T, Sakai G, Ishii H, Hibi T. Improvement of gastrointestinal quality of life scores in cases of Helicobacter pylori-positive functional dyspepsia after successful eradication therapy. J Gastroenterol Hepatol. 2005;20:1652-1660.

10. Suzuki H, Mori M, Kai A, et al. Effect of rebamipide on $H$. pylori-associated gastric mucosal injury in Mongolian gerbils. Dig Dis Sci. 1998;43:181S-187S.

11. Suzuki H, Moayyedi P. Helicobacter pylori infection in functional dyspepsia. Nat Rev Gastroenterol Hepatol. 2013;10: $168-174$.

12. Mirbagheri SA, Khajavirad N, Rakhshani N, Ostovaneh MR, Hoseini SM, Hoseini V. Impact of Helicobacter pylori infection and microscopic duodenal histopathological changes on clinical symptoms of patients with functional dyspepsia. Dig Dis Sci. 2012;57:967-972.

13. Mirbagheri SS, Mirbagheri SA, Nabavizadeh B, et al. Impact of microscopic duodenitis on symptomatic response to Helicobacter pylori eradication in functional dyspepsia. Dig Dis Sci. 2015;60: 163-167.

14. Suzuki H, Nishizawa T, Hibi T. Can Helicobacter pylori-associated dyspepsia be categorized as functional dyspepsia? J Gastroenterol Hepatol. 2011;26:42-45.

15. Suzuki H, Matsuzaki J, Hibi T. What is the difference between Helicobacter pylori-associated dyspepsia and functional dyspepsia? J Neurogastroenterol Motil. 2011;17:124-130. 